spectroscopy, which has also proved to be a powerful tool, is described. Finally, details are given of all known experimental results on nuclear moments and spins and their regularities, and the significance is discussed. Prof. Ramsey also contributes a section on nuclear two-body problems and elements of nuclear structure. In this section the experimental evidence for the nature of nuclear forces is discussed. Results on proton-proton and proton-neutron scattering are surveyed for energies up to $400 \mathrm{MeV}$., and an account is given of the theories of scattering and the meson theory of nuclear forces. Finally, the author gives a brief description of the theory of nuclear shell structure.

Prof. K. T. Bainbridge contributes a section on charged particle dynamics and optics, relative isotopic abundances of the elements and atomic masses. The general theory of mass spectrometers is given, including a comprehensive treatment of the different methods of magnetic focusing. Typical mass spectrometers including those of Dempster and Nier and Mattauch are discussed. The section concludes with tables of the relative isotopic abundances of the elements and isotopic masses.

A survey is given by Prof. P. Morrison of the vast amount of experimental work on nuclear reactions which has been carried out during the past decade. The theories of nuclear reactions and their relation to nuclear structure are also thoroughly discussed. A chapter is devoted to nuclear fission and another to nuclear resctions of high energy. Prof. B. T. Feld contributes 380 pages on neutron physies, including a bibliography of sixteen pages. The interaction of neutrons of energy from thermal energies up to many millions of volts is discussed. Experimental techniques of neutron physics are described, and finally the interaction of neutrons with matter in bulk is dealt with, including a chapter on elementary reactor theory.

These two volumes present an encyclopædic survey of the present state of nuclear physics, and should be in the library of all laboratories working on this subject.

J. D. COCKCROFT

\section{RADIOISOTOPE TECHNIQUES IN DIAGNOSIS, THERAPY AND RESEARCH}

Radioisotope Techniques

Proceedings of the Isotope Techniques Conference, Oxford, July 1951, sponsored by the Atomic Energy Research Establishment. Vol. 1: Medical and Physiological Applications. Pp. vi $+466+42$ plates. (London: H.M. Stationery Office, 1953.) $50 s$. net.

GINCE the construction of the low-energy experi$\$$ mental pile (GLEEP) and its suceessor (BEPO) at Harwell, the Atomic Energy Research Establishments at Harwell and at Amersham have supplied radioactive isotopes for clinical and research purposes in Great Britain and in many overseas countries. These Establishments have become, in consequence, informal clearing-houses for problems and ideas relating to the production, manipulation and application of radioactive isotopes. For the steadily increasing number of medical and scientific workers and engineers using radioisotopes, it was therefore highly profitable that senior members of the Estab- lishments should organize a conference on radioisotope techniques in 1951. The conference was held in Oxford and was attended by nearly five hundred representatives of some twenty nations. The proceedings of the conference have been published in two volumes, the first and larger of which, dealing with medical and physiological applications, is reviewed here.

The proceedings contain seventy-one papers together with the discussions which followed their presentation in conference and deal with a very wide range of techniques and applications. The papers are grouped under three main headings: therapy and diagnosis ; biochemistry and metabolic studies; and plant biochemistry. Papers grouped under the first heading deal mainly with the use of iodine-131 in the diagnosis and treatment of abnormal thyroid function, and the use of such isotopes as phosphorus32 , cobalt-60, and cerium-144 as beta- and gammaray sources in irradiation therapy. Papers under the second heading deal with the use of radioisotopes in biochemical and physiological research and cover such subjects as the use of 'labelled' proteins in immunochemistry and the determination of sodium and potassium in invertebrate nerve fibres by activation analysis. The third and smallest group of papers deals mainly with the uptake and distribution of phosphorus-32 in plants either as fertilizer or as labelled insecticides. The discussions were frank and constructive, and in some cases constituted a salutary addendum to the preceding paper.

The papers dealing with the medical applications of radioisotopes in diagnosis and therapy suggest that much has yet to be learned about the use of pile-produced radioisotopes for the selective destruction of tumours in vivo. As a diagnostic aid and also in small doses for the clinical appraisal of metabolic behaviour, radioisotopes appear to be well established with considerable potentialities. The papers illustrate clearly that, in biochemical, physiological and agricultural research, radioisotope techniques have constituted a major advance in recent years : for example, in the study of precursorproduct relations in steady-state systems-an important problem scarcely soluble except by isotope tracer techniques. Many useful and novel techniques are described for the location of radioisotopes in large animals, in plants, on paper chromatograms and in histological sections. Some of the complications of radioisotope applications also receive attention, such as the difficulties of constructing directional counters, collimating powerful gammaray sources and the importance of recognizing radiation effects in labelled fertilizer studies.

The standard of the papers is high for a conference of this sort, when sometimes papers are thinly disguised excuses for participation at a conference. Surprisingly few typographical errors have been noticed. Two papers mysteriously appear in the wrong section (pp. 379 and 381), and the title "Chromatographic Techniques" includes a curious range of subjects. The proceedings provide a unique review of recent developments in radioisotope applications not to be found in current text-books on the subject. Moreover, they provide an indication of future developments, by whom and where, which is of particular value to the radioisotope specialist. Although a more recent conference on radioisotope techniques was held in 1954, it seems unlikely to vitiate the value of the published proceedings of the 1951 conference. F. P. W. WINTERINGHaM 